\title{
Transatlantica
}

Revue d'études américaines. American Studies Journal

\section{« Little Wise Men : Three Friends and the Public Administration World They Made »}

Le système circulatoire des savoirs de gouvernement aux lendemains de la seconde guerre mondiale

\section{Pierre-Yves Saunier}

\section{(2) OpenEdition}

\section{Journals}

Édition électronique

URL : http://journals.openedition.org/transatlantica/1391

DOI : 10.4000/transatlantica.1391

ISSN : 1765-2766

Éditeur

AFEA

Référence électronique

Pierre-Yves Saunier, « «Little Wise Men : Three Friends and the Public Administration World They Made » », Transatlantica [En ligne], 1 | 2007, mis en ligne le 14 mai 2008, consulté le 29 avril 2021. URL : http://journals.openedition.org/transatlantica/1391 ; DOI : https://doi.org/10.4000/ transatlantica.1391

Ce document a été généré automatiquement le 29 avril 2021.

\section{cc) (i) $\odot \odot$}

Transatlantica - Revue d'études américaines est mis à disposition selon les termes de la licence Creative Commons Attribution - Pas d'Utilisation Commerciale - Pas de Modification 4.0 International. 


\section{« Little Wise Men : Three Friends and the Public Administration World They Made »}

Le système circulatoire des savoirs de gouvernement aux lendemains de la seconde guerre mondiale

Pierre-Yves Saunier

Comme les protagonistes de l'ouvrage de Walter Isaacson et Evan Thomas dont le titre est paraphrasé dans l'intitulé de cet article ${ }^{1}$, les personnages de cet article participent à la création d'un monde. S'il ne s'agit pas ici d'une géopolitique nouvelle, avec des acteurs, des équilibres et des clivages inédits, comme le monde de la Guerre Froide dont Dean Acheson et ses amis tentèrent de saisir le développement, on a pourtant bien affaire à une définition inédite de la géographie, de l'organisation et du fonctionnement d'une sphère transnationale consacrée à la discussion et à la mise en forme des questions de gouvernement. L'établissement et la maintenance de cet espace social de définition et de diffusion des savoirs de gouvernement n'est pas du ressort exclusif des protagonistes étudiés dans cet article, ni de la période chronologique sur laquelle il s'attarde. Herbert Emmerich, Charles Ascher et Donald Stone développent leur action sur un terrain qui, de l'essor des sciences camérales en Europe à l'époque moderne jusqu'aux projections impériales des savoirs et schémas administratifs français et britanniques au $19^{\text {ème }}$ siècle, s'inscrit dans la durée. Néanmoins, les deux décennies qui suivent la fin de la Second Guerre Mondiale voient s'ébaucher ce qui est devenu un marché particulièrement actif et assez bien étudié ${ }^{2}$. Aujourd'hui, les entreprises de transfert de politiques publiques et de compétences administratives sont familières, rangées sous les bannières de la lutte contre la corruption, de la création d'agences administratives autonomes, ou, après l'avoir été dans les années 1980-1990 sous les couleurs de la transition démocratique, de la privatisation de certains secteurs, du dégraissage de l'Etat et de la bonne gouvernance. Entre les années 1950 et notre temps les 'valeurs' de cet espace ont donc bien changé (du planning à la dérégulation, de l'administration au contrat, du gouvernement à la gouvernance). Les espaces de l'échange ne sont pas non plus restés stables (avec par exemple l'ouverture d'un bassin 
de circulation des savoirs de gouvernement dans l'ancien bloc soviétique après 1989) ${ }^{3}$. D'autres partenaires interviennent désormais, notamment les grands cabinets d'audit privés. Mais ce sont les structures, la morphologie de cet espace, voir sa polarité qui sont restés profondément marqués par le travail de création de nos trois 'petits sages' dans les années 1940-1960. C'est alors que se solidifie un espace de circulations centré sur les Etats-Unis, marqué par une intégration entre organismes privés (Fondations philanthropiques, universités), organismes inter-gouvernementaux, gouvernements nationaux et organismes non gouvernementaux. Cet article propose de suivre trois des protagonistes de cette phase de mise en place, dans leurs trajectoires et dans leurs pratiques de travail, à travers des documents (correspondances, rapports, agendas...) qui permettent de saisir leur travail de l'intérieur ${ }^{4}$. Pour mieux saisir ce qu'ils mettent en œuvre, pour mieux comprendre au nom de quoi ils parlent, pour faire voir ce qu'ils peuvent porter sur la scène transnationale, il faut commencer par une double visite, géographique et thématique, sur les terrains de la public administration états-unienne, cette branche de la science politique qui se pose en savoir de gouvernement.

Le système de la public administration aux Etats-Unis d'Amérique

2 Perri Arnold, dans son ouvrage sur la réorganisation de la branche présidentielle de l'exécutif états-unien, a employé l'expression de " public administration establishment " pour décrire la forme prise dans ce pays par un ensemble d'hommes et d'institutions qui, de la fin des années 1920 aux années 1950 environ, font exister la public administration comme discipline universitaire, comme corps de principes, comme communauté de savants et de praticiens et comme registre d'action ${ }^{5}$. Ce qui frappe peut être encore plus que l'existence d'un groupe fermé contrôlant l'accès à l'espace social et théorique de la public administration, c'est l'intégration systématique d'acteurs et d'organisations aux origines communes dans un projet d'action et de coordination pour «l'amélioration du gouvernement ». Cette intégration intellectuelle et pratique est au cœur de l'interrogation d'un certain nombre d'ouvrages depuis le milieu des années 1980, en un débat sur l'histoire de l'Etat américain ${ }^{6}$.

3 Ce système se construit sur les divers ordres de pratiques ébauchés depuis les années 1890, lorsque Woodrow Wilson et Franck Goodnow définirent l'étude de l'administration comme un champ pour la science politique, en un mouvement qui unissait quête d'émancipation disciplinaire (par rapport au droit), souci d'action politique (dans le cadre des mouvements de réforme) et réflexion philosophique (autour de la redéfinition de la place et du rôle du citoyen et du pouvoir) ${ }^{7}$. Cette pluralité d'objectifs, de principes et d'acteurs contribua à délimiter un espace disputé, où s'allièrent et se désunirent des définitions et des contenus portés par des groupes divers, des universitaires en quête d'affirmation disciplinaire aux militantes des maisons sociales (settlement houses) plaidant pour une administration municipale fournisseuse de services de qualité, en passant par les politiciens réformateurs soucieux de limiter les effets des machines politiques, les partisans de la rationalisation taylorienne du travail administratif, et ceux pour qui une administration efficace et rationnelle était la condition de l'avènement d'une nouvelle citoyenneté participative et responsable ${ }^{8}$.

4 C'est finalement une définition très technique de l'étude de l'administration qui émerge de cette bataille. L'icône de ce premier moment de l'étude de l'administration est sans doute la mise en place de la procédure du budget, commencée dans les municipalités, poursuivie dans les états et poussée jusqu'au niveau fédéral ${ }^{9}$. Dans l'ensemble, l'étude 
de l'administration est alors soucieuse de machinerie administrative (spécialisation fonctionnelle des services), de mise en ordre des dépenses et des recettes, de lignes de commandement ou des modalités de recrutement et de gestion du personnel administratif (civil service reform). Ces préoccupations dominent dans les travaux de conseil aux administrations de l'Institute for Government Research ou du National Institute of Public Administration, et s'incarnent dans la figure de Frederick Cleveland, ancien professeur de finance, spécialiste du budget et expert majeur des premières décennies du siècle.

Un premier ensemble d'institutions naquit de ces prémices chahutés, sous la forme de commissions d'études ponctuelles (la Commission on Economy and Efficiency nommée par le Président Taft en 1910), de formations universitaires (l'University of Michigan crée un diplôme supérieur en administration municipale en 1914), de traités académiques (Politics and administration de Goodnow en 1900), ou d'agences d'études (création du New York Bureau of Municipal Research en 1907). Cette première configuration de l'étude de l'administration est néanmoins marquée par la fragilité institutionnelle, et par une triple marginalité puisqu'elle géographiquement centrée sur la ville de New York, thématiquement orientée sur l'administration et le gouvernement municipal et politiquement marquée une connotation militante réformiste. Les années 1920 voient émerger une nouvelle configuration marquée par la mise en système des institutions existantes, la mise en synergie des universitaires et des administrateurs et la volonté claire de mettre la science sociale science au service des choix politiques en une nouvelle configuration objective et neutre de l'étude de l'administration. La public administration, vocable dont l'usage se généralise alors, est mise en traité par Leonard White, premier titulaire d'une chaire en public administration, dans son Introduction to the study of public administration, premier manuel de la discipline émergente (1926). Il y postule l'unité du processus administratif, l'émancipation de la nouvelle discipline par rapport au droit et son lien théorique avec les bases du management, la possibilité d'une administration scientifique et la centralité de l'administration dans la question du gouvernement contemporain. Ce nouveau système est initié par des hommes familiers des institutions de la première génération des institutions de l'étude de l'administration pour les avoir animées, soutenues ou créées. Il résulte d'une coalition stratégique entre universitaires (Leonard White et Charles Merriam, du département de science politique de University of Chicago), experts du conseil et de la réforme administrative (Luther Gulick, directeur du National Institute of Public Administration), administrateurs locaux, d'états ou fédéraux (Louis Brownlow, directeur de services municipaux-city manager) et gestionnaires des grandes fondations philanthropiques (Raymond Fosdick et Beardsley Ruml, des responsables du complexe philanthropique rockefellerien) ${ }^{10}$. Tous ceux là vont se trouver associés à la création et à la gestion des nouvelles institutions qui forment le système de la public administration. On les retrouve à partir de 1929-1930 autour de la Public Administration Clearing House et du 1313 center, groupement d'associations professionnelles d'administrateurs et d'élus de tous secteurs et de tous niveaux dont la mission est de contribuer à 'l'amélioration du gouvernement' par des actions directes dans l'administration et auprès des administrateurs (formation, services de conseil, recherche, standardisation des procédures, organisation de la profession...). ${ }^{11}$ Ce sont aussi ces hommes qui chapeautent l'essentiel du travail de recherche en public administration, à partir du Committee on Public Administration créé en 1929 au sein du Social Science Research Council. On les retrouve encore dans 
l'animation des formations universitaires en public administration, qu'il s'agisse du programme mis en place à partir du milieu des années 1920 à University of Chicago ou de la Maxwell School of Citizenship and Public Affairs de Syracuse University. Ce sont eux qui animent l'American Society of Public Administration fondée en 1939, son journal Public Administration Review et les divers comités qui assurent la vie scientifique de la communauté. Enfin, ils travaillent en étroite association avec l'organisation qui finance et coordonne le système, le Spelman Fund of New York, organe de la philanthropie Rockefeller créé en 1929 et voué à partir de 1930 à l'activité d'organisation du champ de la public administration. Du « méridien » de ce système, qui passe désormais par le campus de University of Chicago, en une incarnation recherchée de la distance vis à vis des centres du pouvoir de la Côte Est, émanent alors toute une série d'initiatives qui changent le fonctionnement organique, académique et pratique de l'administration états-unienne. Le travail en profondeur auprès des administrateurs eux mêmes produit des résultats aussi divers que l'augmentation du niveau de diplôme des recrues, la standardisation des procédures administratives, le développement d'un esprit de corps ou la circulation géographique accrue des administrateurs. La public administration devient dans le même temps un secteur spécifique d'activité universitaire, tant sur le versant de l'enseignement que sur celui de la recherche, alors que la version savante de la public administration insiste de plus en plus, dans ses traités, sur le contexte social et politique de l'activité administrative, dont les fonctions de programmation et de planification sont mises en avant. La public administration comme corps de savoir et comme pratique est alors très liée à l'extension des activités gouvernementales dans les domaines des politiques sociales et économiques, notamment au niveau du fédéral, tant durant le New Deal que durant les années de la Seconde Guerre Mondiale. Les administrations elles-mêmes voient leurs pratiques quotidiennes modifiées. Des changements prennent place au sein des municipalités, notamment suite aux missions d'audit administratif et de conseil en amélioration organisationnelle de Public Administration Service, l'agence de conseil créée par les diverses associations du 1313 au milieu des années 1930, ou suite au travail des travailleurs de terrain des diverses associations. Ils se développent aussi au cœur des administrations de certains états dont les structures sont réorganisées avec les conseils des hommes du 1313 et les financements du Spelman Fund. Ils s'incarnent enfin dans les nouvelles agences créées lors du New Deal (notamment dans les secteurs du logement, des politiques sociales, de la planification urbaine et régionale) dont l'administration fait appel aux compétences des hommes du 1313, et jusqu'en haut du gouvernement fédéral dont la branche exécutive est réorganisée en 1939 selon des lignes tracées par une commission dirigée par Louis Brownlow ${ }^{12}$. Le Bureau of the Budget, une des créations principales du plan de réorganisation de l'exécutif présidentiel mis en œuvre en avril 1939, destiné à renforcer les moyens d'action, de programmation et de contrôle du Président des Etats-Unis et qui va être un des principaux organes administratifs de la présidence jusqu'aux années 1960, est une des illustrations de cet impact de l'action du système de la public administration. Il n'est pas indifférent que les premières équipes qui l'animent soient très liées au 1313 center, qu'il s'agisse des membres du noyau dur de son équipe, choisis au sein de l'équipe de Public Administration Service, ou de son premier directeur Harold Smith, un proche de Louis Brownlow $^{13}$. Enfin, c'est dans l'intégration des différents niveaux de gouvernement (local, étatique, fédéral), ce qu'on appelle les intergovernmental relations, que l'impact du système mis en place dans les années 1930 est peut être le plus fort et le plus durable, 
en ce qu'il repose sur une utilisation de toutes les possibilités d'intégration offertes par les ressources disponibles: par les pratiques, par les acteurs, par la recherche, par l'enseignement, par le conseil ${ }^{14}$.

6 Les réseaux, les structures et les concepts mis en place au cours de la décennie 1930 semblent s'effriter à partir de la fin des années 1950 sur la scène domestique. Des éléments du système disparaissent alors : le Spelman Fund of New York met fin à ses activités en 1946, la Public Administration Clearing House disparaît en 1956, le Public Administration Committee du Social Science Research Council ferme lui aussi boutique. Dans le même temps, de nouveaux acteurs apparaissent sur la scène, qu'il s'agisse de lieux de recherche et d'enseignement (Harvard School of Government) ou d'auteurs (Dwight Valdo, Herbert Simon, Robert Dahl) qui remettent en cause certains des canons de la version savante de la public administration, notamment à l'occasion de la vague behaviouriste en sciences sociales. Mais la plupart de ces nouvelles données s'intègrent aux espaces théoriques et sociaux du système né dans les années 1930, d'autant que les organisations savantes et professionnelles créées à ce moment continuent leur action et assurent une pérennité de principes, de normes et de pratiques.

Public administration, sciences administratives, sciences de gouvernement et circulations

Cette public administration états-unienne est une des versions du rapport entre savoir et action politique et administrative. Ce rapport a une histoire longue, fortement liée à celle des Etats, histoire qui met en scène de nombreux protagonistes, depuis les juristes des Rois de France aux philosophes de cours en passant par les caméralistes allemands, et qui s'enrichit considérablement de pratiques de dépaysement en direction des espaces extra-occidentaux (notamment vers le monde chinois) ${ }^{15}$. Inscrire le moment contemporain dans cette histoire longue demanderait plus que l'invocation des mânes d'Auguste Comte ou de Saint Simon dans De la réorganisation de la société européenne, et en tracer l'identification progressive comme une conception spécifique de la politique amènerait sans doute au portrait de changements très progressifs et non linéaires dans la conception de ce qu'est le "gouvernement ", changements dont l'onde dépasse sans doute les scansions canoniques de l'histoire. Cette préoccupation fournit en ce moment même l'armature à de nombreuses recherches dont témoignent plusieurs publications récentes ${ }^{16}$. Un des traits fondamentaux du moment contemporain semble être la problématisation scientifique des actes de gouvernement et d'administration, problématisation qui se traduit par un intérêt marqué pour l'administration comme branche apolitique, et donc objectivable, du gouvernement. Cette évolution vers une mise en science semble particulièrement marquée à partir des dernières décennies du $19^{\text {ème }}$ siècle, dans des sociétés européennes et nord-américaines aux prises avec les constats de mutations sociales, politiques, culturelles que différents protagonistes identifient comme appelant des modalités nouvelles de contrôle et de régulation. Dès lors, contrôle social, ordre social, progrès social peuvent rimer avec perfectionnement de l'outil administratif et gouvernemental.

8 «Les procédés légaux de l'administration remplaceront de plus en plus les procédés arbitraires du gouvernement; ou si l'on veut le gouvernement ne sera plus comme le concevait Rousseau qu'une suprême administration $»^{17}$. "Je pense que la civilisation elle-même dépend de notre habileté à développer la Science, la philosophie et la pratique de l'Administration capable d'accomplir les fonctions publiques de la société civilisée ${ }^{18}$. Il serait assez facile de fournir une longue liste de citations, en provenance des Etats-Unis d'Amérique ou d'Europe, qui témoigneraient d'un état d'esprit 
semblable, propension à insister sur l'aspect administratif de l'activité de gouvernement d'une part, et présentation de l'administration comme objet de science d'autre part. Plusieurs avatars de cette 'mise en science' sont aisément repérables au tournant entre $19^{\text {ème }}$ et $20^{\text {ème }^{2}}$ siècle ${ }^{19}$. Leurs variations concernent les approches, qui couvrent une gamme allant de l'approche par l'ingénierie et la science de l'organisation ${ }^{20}$ jusqu'à la problématisation par les sciences sociales ${ }^{21}$ en passant par le renforcement $d u$ lien entre travail administratif et travail juridique ${ }^{22}$. Ces variations concernent aussi les groupes sociaux, politiques et professionnels mobilisés, ou les différents niveaux de gouvernement et d'administration qui sont appréhendés, ou encore l'intensité variable avec laquelle les gouvernements et les administrateurs se mêlent à l'entreprise ${ }^{23}$. La public administration états-unienne est un de ces avatars.

9 Une autre caractéristique du moment contemporain réside dans l'internationalisation précoce des confrontations, rapprochements, échanges entre ces diverses approches, alliances, groupes et définitions. Cette internationalisation, dont on a proposé de définir le stade actuel en parlant de "marchés internationaux de la réforme des institutions d'Etat $\aleph^{24}$, me semble même constitutive même des différentes positions, à l'image de ce qui se produit autour d'autres thèmes tous liés à de semblables réflexions autour des nouvelles formes du contrôle et de l'ordre social ${ }^{25}$. En ce qui nous concerne, elle prend forme comme structure d'échange entre administrateurs et universitaires autour des thèmes de l'administration et du gouvernement. Elle se développe par le truchement de séjours à l'étranger (voyages, études, visites), de correspondances, de publications savantes (notamment dans les colonnes des périodiques), de traductions d'ouvrages, de conférences, touchant à la fois des administrateurs, des élus, des réformateurs amateurs mais aussi et de manière croissante des spécialistes universitaires en provenance du droit ou des nouvelles disciplines en cours de construction. Ce versant 'volontariste' de la structure d'échange est assez précocement structuré par les activités et les réseaux de diverses associations inscrites dans le milieu du droit (notamment celles qui s'occupent d'arbitrage des conflits internationaux ou encore les premiers groupements savants de droit international et comparé). Les Congrès Internationaux des Sciences Administratives à partir de 1910, et l'Institut International des Sciences Administratives créé à l'issue du Congrès de 1930 sont une des structures associatives qui organise et formalise ces échanges ${ }^{26}$. Il faut souligner que l'échange peut avoir lieu sous des formes bien plus contraintes, souvent en combinaison avec les formes précédentes. Gouvernements nationaux et organismes privés participent très tôt à ce commerce international des pratiques et principes de l'administration et du gouvernement. Au sein des empires coloniaux, dans le cadre de rapports de domination ou entre nations alliées prennent place des flux de conseils et de missions. Les travaux menés au sein de la Pan-American Union ou dans les pays d'Amérique Latine et des Caraïbes par les leaders états-uniens de l'étude de l'administration, les missions en Chine d'un certain nombre d'experts administratifs états-uniens et européens en témoignent parmi de nombreux autres cas qui semblent se multiplier au tournant des $19^{\text {ème }}$ et $20^{\text {ème }}$ siècle $^{27}$. Un marché international s'ébauche même dans des bassins précis, comme par exemple l'Amérique du Nord, ou les firmes d'ingénierie administrative de Chicago, nées du mouvement de réforme municipale des premières années du siècle, commencent à travailler au Canada ${ }^{28}$.

10 Les grandes fondations philanthropiques américaines, qui s'intéressent de manière suivie au gouvernement et à l'administration à partir du début des années 1930, contribuent alors à structurer ces échanges internationaux. Le Spelman Fund of New 
York dans l'entre deux guerres, les programmes de la Fondation Ford à partir des années 1950, participent en même temps de la réorganisation des flux transatlantiques et globaux. Jusqu'aux années 1930, conseils, paradigmes hommes et livres circulent plutôt, quoique non uniquement, de l'Europe vers les Etats-Unis. L'Europe des protagonistes états-uniens de l'étude de l'administration est alors un espace de ressources rhétoriques et pratiques, un réservoir d'exhortations et de recettes. A partir des années 1940, le flux s'inverse et le système de la public administration états-unienne part à la conquête du monde. La pax americana y est pour beaucoup, avec les procédures d'exportation que secrètent ses divers programmes, du Plan Marshall au Point IV Program de Truman. L'Europe devient alors un espace de pratiques pour les acteurs de la public administration, tout comme les nouveaux pays nés de la décolonisation. Le travail domestique et les liens internationaux développés dans les années 1930 par le système états-unien de la public administration évoqué ci-dessus sont une autre des conditions de l'efficacité de cette inversion géopolitique des flux. Les spécialistes universitaires et privés de la public administration, à travers les réseaux savants, par le biais des programmes d'assistance technique du gouvernement états-unien ou au cœur des organismes spécialisés des grands organismes internationaux, assurent alors à la public administration un développement universel qui se traduit notamment par la floraison d'écoles et d'associations de Publica Amministracion/ Public Administration/ Administration publique à travers l'Amérique Latine, l'Asie ou l'Afrique, avec le soutien de la Fondation Ford, qui finance de nombreux programmes d'éducation, de recherche et de conseil en public administration dans les années 1950-1960 ${ }^{29}$. L'idiome partagé de ces nouvelles organisations, et l'approche du gouvernement et de l'administration dans les années 1950-1960 est très largement celui de la public administration formalisée et organisée dans les années 1930, agrémentée de ses nouvelles trouvailles techniques (policy analysis, PPBS...) qui sont largement exportées, adoptées ou adaptées ${ }^{30}$. Charles Ascher, Herbert Emmerich et Donald Stone sont trois figures éminentes de cette réorganisation des flux transnationaux en matière de public administration. Il n'est pas question ici de présenter en détail leur activité, mais plutôt de proposer quelques ouvertures et pistes au sujet de leur travail quotidien d'internationalisation. L'activité et les caractéristiques de Charles Ascher, d'Herbert Emmerich et de Donald Stone les posent en démiurges et praticiens consommé de ces processus, dont la finalité explicite est dans leur cas de produire des objets universels, ayant cours sur l'ensemble de la planète, qu'il s'agisse de structures associatives, de formes d'organisation, de principes d'action ou de concepts et de pratiques dans la sphère de l'organisation de l'administration publique. L'activité d'Ascher et des autres membres du régime transnational de la public administration s'inscrit en effet dans un projet universaliste et universalisant, qui postule l'existence de phénomènes identifiables comme relevant de l'activité d'administration et susceptibles d'être appréhendés d'une semblable manière scientifique quel que soit leur contexte, même s'il faut noter la grande flexibilité des praticiens de la public administration en termes de réalisations pratiques et leur disponibilité pour les adaptations aux systèmes locaux.

Charles Ascher, le Mickey Mouse de la public administration

11 Ce sous titre se réfère à une métaphore employée par Charles S. Ascher, un des personnages les plus actifs rencontré lors de mes travaux sur l'histoire contemporaine des circulations et connexions internationales autour du terrain urbain et des structures d'administration et de gouvernement ${ }^{31}$ : 
J'ai passé plusieurs heures avec Tickner, Emmerich et Emil Sady sur le brouillon d'une lettre de la Public Administration Branch de l'United Nations Technical Assistance Administration invitant l'International Union of Local Authorities à proposer une étude de la fourniture de services aux gouvernements locaux par les gouvernements nationaux ou les Unions de villes, afin de stimuler l'activité sur ces thèmes dans les pays sous développés. Maintenant, tel Mickey Mouse jouant au tennis, je me précipite de l'autre côté du filet pour aider à retourner le service. J'ai appris qu'Emmerich serait à la table ronde de l'International Institute of Administrative Sciences en Belgique, et je le pousse à venir à La Haye pour rencontrer Arkema [le secrétaire de l'International Union of Local Authorities] afin de précipiter les choses lors d'une négociation directe en juillet prochain ${ }^{32}$.

La métaphore, tout autant que les évènements relatés ou le document dont elle est extraite (un bulletin d'information - round up - de Ascher), est fidèle aux activités de celui qui fut l'un des protagonistes principaux d'un projet d'internationalisation de la public administration comme discipline, pratique, théorie et communauté entre les années 1930 et les années 1960.

Le travail quotidien d'Ascher entre la fin des années 1940 et la fin des années 1960 permet de considérer dans le détail les méthodes, possibilités et contraintes contenues dans la position qui est la sienne, posture que l'on pourrait peut être capturer sous l'appellation $\mathrm{d}$ ' " interprète de l'universel », en dérivant cette notion des propositions de Pascale Casanova autour de la «fabrique de l'universel» dans l'espace littéraire et du rôle des intermédiaires de la "traduction» au sens large du terme ${ }^{33}$. Cette appellation correspondrait à la posture adoptée par Ascher dans toute son activité, où il prend soin de ne jamais prêter flanc à aucune suspicion quant à ses ambitions personnelles ou quant à sa soumission à des stratégies nationales, toujours soucieux d'être un recours, une force de proposition «neutre». Elle correspondrait aussi à l'ordre de légitimité et de validité dont Ascher se réclame pour justifier ses propositions, ordre supérieur défini à la convergence d'une part des règles de l'objectivité scientifique dans l'approche des phénomènes administratifs, des méthodes de gouvernement ou des problématiques de politique publique, d'autre part des espérances et projets pour des formes de société planétaire unifiée qui sont le référent ultime des divers projets 'globalisants' sous leurs diverses espèces ${ }^{34}$. La notion d'interprète rend encore compte du travail de traduction et d'adaptation que Ascher effectue sans cesse pour rendre possible les interactions entre les différents partis qu'il met en contact, et la multiplicité de ses fonctions pratiques (passeur actif, truchement, introducteur, organisateur, éminence grise, manipulateur, prescripteur...). Enfin, elle rappelle que son activité se situe dans des limites et s'exerce sous des contraintes qui ne sont pas ici linguistiques ou littéraires mais s'inscrivent dans un contexte de géopolitique savante et diplomatique, à la jonction et sous le rayonnement d'espaces sociaux et politiques aussi divers que les batailles de couloir onusiennes, les jeux du monde académique, les relations diplomatiques bilatérales et multilatérales, les perceptions culturelles transatlantiques ou transhémisphériques, les problèmes concrets de l'administration des pays d'Amérique Latine ou d'Asie ou les mutations des systèmes de pouvoir aux Etats-Unis (alternances politiques, modifications structurelles des caractéristiques sociales et culturelles des responsables administratifs et politiques...).

14 Figure de second rang, absent ou marginal dans les index des ouvrages de science politique ou de public administration, Ascher est pourtant au centre des réseaux étatsuniens et internationaux de la public administration, réseaux qu'il met en place et fait 
vivre dans le long terme avec ses partenaires états-uniens, européens, asiatiques, latino-américains. En jouant sur les mots, on peut le définir comme un clerc (intellectuel) et comme un clerk (employé de bureau, vendeur) de la public administration. Les bases de son action internationale et internationalisante sont posées dans les années 1930, lorsqu'il rejoint le 1313 center. La participation d'Ascher à ce complexe met une fin à sa carrière de juriste, commencée en 1921 après l'obtention d'un Ll.B de droit à Columbia University ${ }^{35}$. Assistant du directeur de la Public Administration Clearing House Louis Brownlow, qui coiffe la vingtaine de groupements finalement associés au 1313, il contribue à gérer, organiser, créer des associations d'administrateurs ou d'élus et leurs service communs (documentation, publication, audit, conseil, ingénierie...). Il y apprend le métier de l'administration des associations et de leurs activités (organisation de conférences, réunions, rencontres), de la coordination inter-associative, de la mise en synergie entre associations, gouvernements et monde de la recherche en sciences sociales, du développement et de l'activation de réseaux, de la définition et circulation de l'information, ainsi que la routine grise et minutieuse des travaux d'enregistrement des décisions, des noms des personnes et des lieux, des circonstances ou le travail incertain et incessant des relations humaines dans des situations de coordination non-hiérarchiques. C'est tout ce B.A-BA non formalisé qui lui permet, avec ses complices Louis Brownlow, Don Price, Donald Stone, Herbert Emmerich, et quelques autres, une maitrise sociale, théorique et culturelle de la public administration états-unienne dans les années 1930. Il y conquiert aussi peut être cette "passion de l'anonymat ", maîtrise paradoxale de l'effacement actif et de la discrétion agissante que son mentor Louis Brownlow établit en condition d'une action efficace dans la sphère de l'amélioration des pratiques gouvernementales et administratives ${ }^{36}$. C'est aussi par cette association avec le 1313 qu'Ascher constitue la ressource domestique sociale et intellectuelle de son action internationale à venir. Le réseau des associations du 1313, qui inclut aussi bien les gouverneurs d'états que les responsables des départements fiscaux des municipalités ou les fonctionnaires fédéraux des politiques sociales, le place au contact des pratiques et innovations administratives, tout particulièrement avec ces structures et pratiques nouvelles que le New Deal fait fleurir dans les Etats-Unis des années 1930 : agences semi autonomes et spécialisées, politiques sectorielles et administrations nouvelles (logement, sécurité sociale...), service de recherche en management du Bureau of Budget, réorganisation de l'exécutif fédéral.... Le programme international du 1313, marqué par une activité d'importation des pratiques administratives européennes et une implication croissante dans le travail et le fonctionnement de certaines associations internationales jugées propices à soutenir cette activité importatrice (International Federation for Housing and Town Planning, International Union of Local Authorities, International Institute of Administrative Sciences), participe aussi à l'inscription d'Ascher dans un cercle plus large, même s'il ne prend pas alors part directement aux flux transatlantiques (congrès, voyage d'études, comités de direction des associations internationales) qui sont une composante capitale de la conception et du programme du 1313. Cette activité le place enfin au cœur des contacts interindividuels qui font exister le petit monde états-unien de la public administration, dans les sphères gouvernementales, les milieux universitaires ou les institutions privées de la recherche gouvernementale. C'est d'ailleurs en tant que secrétaire de la branche recherche du Public Administration Committee du Social Science Research Council, la structure qui organise la recherche en sciences sociales dans les Etats-Unis de l'entre deux guerres, qu'Ascher fait ses 
premiers pas personnels sur le terrain de l'internationalisation, avec l'organisation de deux conférences sur l'administration des organisations internationales et l'organisation supranationale ${ }^{37}$. Il bascule définitivement dans cette dimension au lendemain de la Seconde Guerre mondiale. Assistant du directeur général de l'UNESCO d'avril 1947 à décembre 1948, chargé de mettre en place le programme de l'organisme, représentant officiel des associations internationales liées à la Public Administration Clearing House auprès des Nations Unies et des autres organismes internationaux (à partir de 1949), puis co-directeur et directeur du centre international que Public Aadministration Clearing House créé à New York à quelques encablures du siège des Nations-Unies (à partir de 1950), Ascher commence alors le travail qui l'occupe jusqu'au début des années 1970. Pendant ce quart de siècle, Ascher est une des chevilles ouvrières de la mise en place et du fonctionnement d'un système international en public administration. Ce système, propulsé par les financements de la grande philanthropie états-unienne, met en relation des associations internationales, des organismes intergouvernementaux, des structures états-uniennes (sociétés d'administrateurs, groupes académiques, agences gouvernementales comme l'International Cooperation Agency/US Agency for International Development ou l'United States Overseas Mission, commissions d'attribution de bourses...), des groupes nationaux ou régionaux (écoles et instituts d'administration, sociétés savantes et professionnelles) et ces mêmes fondations philanthropiques. Il est tourné, pour ceux qui l'animent, vers la perspective lointaine d'un ordre mondial pacifié par la prospérité née de la société de marché et la diffusion de la démocratie libérale, perspective qui appelle le perfectionnement de la machine administrative et gouvernementale, du niveau local jusqu'à l'échelle supranationale. Cette perspective administrative globale, ébauchée dans les années 1930 au 1313, et tout à fait en harmonie avec les visions qui soutiennent l'action de la philanthropie rockefellerienne ou carnegienne depuis le début du $20^{\text {ème }}$ siècle, s'accompagne de perspectives régionales (hémisphériques) de moyen terme, et se traduit dans le court terme par l'investissement dans la mise en relation des différents acteurs qui peuvent participer à l'avènement espéré, en une version sophistiquée des premières considérations carnegiennes sur la compréhension mutuelle (mutual understanding $)^{38}$.

15 Cette configuration ordonnée d'acteurs, que j'ai commence d'explorer, produit des textes théoriques et pratiques en public administration, des rapports, des ouvrages de recherche tout autant que des réformes administratives, des structures de recherche et d'enseignement en public administration ou des structures savantes et sociables d'universitaires et de praticiens. Plus que d'imposer une manière unique de définir les contenus de l'activité administrative et gouvernementale, il diffuse et établit des manières de faire, des valeurs, des cadres de référence assez flexibles pour être accueillis et adaptés à travers l'Europe, l'Asie, l'Afrique ou l'Amérique Latine par des partenaires désireux de participer à cette entreprise tout autant pratique, théorique, politique, sociale et culturelle.

16 Le rôle d'Ascher dépasse la simple fonction de transmission. Il n'est pas qu'un messager de bonne volonté qui fait circuler les informations dans un réseau ou un réseau de réseaux. Sous réserve d'inventaire, et quoique ces succès soient ponctuels et sans cesse à confirmer, il semble avoir contribué par une présence continue et un 'lobbying thématique' de tous les instants à faire rentrer des thèmes comme l'administration, l'urbain et l'urbanisation dans les agendas des organisations inter-gouvernementales avec lesquelles il travaille. Pour ce qui nous concerne ici, et pendant les décennies où il 
a animé l'internationalisation de la public administration avec ses complices de l'ère du 1313 (Donald Stone, Herbert Emmerich, Don Price, Rowland Egger entre autres), Ascher a puissamment contribué à mettre en place les réseaux qui font vivre le système, à créer certaines des structures du système, et à définir les rapports entre les principales structures mises en système. Outre son rôle d'aiguillon auprès des élites administratives que sa propre action et celle de ses compagnons ont contribué à mobiliser et organiser dans diverses régions du monde, et notamment dans les nouvelles nations, ou son rôle d'intermédiaire pour dégager des ressources financières ou humaines pour leurs organisations (bourses, stagiaires, contrats), il a notamment façonné de l'intérieur les associations internationales qu'il représente auprès des organismes internationaux, agissant avec force pour leur transformation en groupements à couverture planétaire et multifonctionnelle (mêlant professionnels et agents gouvernementaux) et en organismes de services pour leurs membres, pour les gouvernements locaux et nationaux et pour les organismes intergouvernementaux. En bref, c'est à la définition pratique de la catégorie 'organisation non gouvernementale' qu'il a ainsi œuvré au cours de plusieurs décennies de liaison entre organisations non gouvernementales et organisations intergouvernementales, redoublées par son activité au sein de la Conference of Non-Governmental Organizations in Consultative Relationship with the United Nations (CONGO). C'est d'ailleurs à ce moment que son association avec son vieil ami Herbert Emmerich est particulièrement intense, du fait de la circulation des informations entre eux et de l'action croisée et concertée qu'ils sont dès lors à même de mener.

Herbert Emmerich, ou l'amitié comme ressource

C'est entre 1910 et 1914 à l'Ethical Culture School ${ }^{39}$ que Herbert Emmerich, né à New York en avril 1897, se lie d'amitié avec Charles Ascher son cadet de deux ans. Leur correspondance se plaît à rappeler cette longue et fidèle relation, même si elle ne revient pas sur d'autres éléments qui rapprochent les deux hommes. Emmerich est en effet, comme Ascher, un enfant du West Side, originaire d'une famille juive allemande d'immigration assez récente, et son père, comme celui d'Ascher, est un petit industriel sans postérité (fabrique de rubans en soie). Emmerich fréquente néanmoins l'école à titre payant, et non en boursier comme Ascher dont le père, fabricant de cigares, a fait faillite. Leurs trajectoires ne divergent qu'un bref moment après la fin de leurs études secondaires. Emmerich effectue un cursus universitaire en économie, à la Wharton School de University of Pennsylvania. Il y reçoit l'enseignement de Simon Patten, un des pères fondateurs de la discipline économique outre Atlantique et de sa filiation avec l'historicisme allemand, dont un Emmerich plus âgé rappellera à ses correspondants les considérations sur l'interdépendance des individus et des sociétés ou les réflexions sur la société d'abondance. Après quelques années erratiques durant lesquelles le jeune Emmerich émarge aux services secrets états-uniens, à une firme de conseil industriel et d'ingénierie managériale, à la firme paternelle et à celle de son beau père lui même petit industriel de la bonneterie, il trouve sa niche à la City Housing Corporation à partir de 1924. Jusqu'en 1933, il est le vice-président de cette société de logement à dividendes limités dirigée par Alexander Bing, parrain de l'Ethical Culture School et ancien président de l'American Ethical Union. La société veut proposer des solutions innovantes aux problèmes du logement et de l'urbanisme et construit les communautés de Sunnyside (dans le Queens new-yorkais) puis de Radburn (New Jersey). Emmerich, Démocrate, rejoint alors le staff de Henry Morgenthau Jr. à la toute nouvelle Farm Credit Administration. Il y joue un rôle majeur dans la rédaction, la négociation et le 
passage du Federal Credit Union Act, mission pour laquelle il est choisi à cause de sa connexion militante avec le mouvement coopératif et de ses origines urbaines 'neutres'. Il administre ensuite l'exécution de ce programme de refinancement et de centralisation des opérations de crédit à l'agriculture états-unienne et finit par présider aux destinées de la Farm Credit Administration elle-même, une des plus considérées parmi les nouvelles agences fédérales. Il renoue avec ses vieux compagnons de Radburn en 1937, lorsque Louis Brownlow, ancien consultant municipal de la City Housing Corporation, lui propose de devenir son bras droit à la direction de la Public Administration Clearing House. Il y remplira un rôle de coordination entre les différentes associations d'administrateurs du 1313 center. Comme bien d'autres membres de ces groupes, son activité de consultant auprès de l'administration fédérale le ramène au sein de celle ci pendant la Seconde Guerre. Tout en maintenant ses liens avec la Public Administration Clearing House, Emmerich occupe des postes de direction à l'Office of Productions Management/ War Production Board (1941-1942), l'organisme qui gère et planifie la production industrielle de guerre aux Etats Unis, puis à la Federal Public Housing Authority qui distribue les fonds aux autorités locales pour la construction de logements publics. Lorsque Louis Brownlow prend sa retraite en 1945, Emmerich revient à Chicago pour lui succéder à la tête de Public Administration Clearing House, et s'inscrit immédiatement dans le nouvel axe d'administration internationale ouvert au 1313 à partir du début des années 1940 (notamment à travers la participation à la conception et gestion administrative de l'United Nations Relief and Rehabilitation Administration, première agence des Nations Unies en 1943). Emmerich fait de cette activité internationale le fer de lance de l'activité du groupe, au détriment de la pérennité qu'il aurait pu lui donner en maintenant son investissement dans les affaires administratives domestiques ${ }^{40}$.

18 A l'instar d'autres spécialistes du 1313, il contribue personnellement à la mise en place des nouveaux organes de l'ordre mondial dans leur dimension administrative. Cela est particulièrement marqué pour l'UNESCO, à la conférence préparatoire de laquelle il assiste comme membre de la délégation états-unienne, et pour laquelle il remplira plusieurs missions d'études sur son organisation administrative ${ }^{41}$. Dans les années qui suivent, son activité de consultant/conseiller est très variée : on le retrouve auprès du Département d'Etat, du gouverneur de Porto-Rico, de la US Atomic Energy Commission, $\mathrm{du}$ Bureau of Budget ou du President's Advisory Committee on Management Improvement. Mais c'est bien autour de l'administration des objets internationaux que son intérêt se précise. Il décroche pour PACH deux bourses de la Fondation Ford en 1950 et 1952 qui ancrent définitivement l'organisation dans l'international ${ }^{42}$. Ces 1,3 millions de $\$^{43}$ permettent de financer un large programme en public administration, dont le but est d'améliorer la capacité des Etats-Unis à administrer et de coordonner leurs politiques domestiques et internationales d'une part, et d'améliorer l'administration des organisations intergouvernementales d'autre part. La création d'un bureau de Public Administration Clearing House à New York, pour donner assistance et conseil aux Nations Unies, à leurs agences et aux Organisations non gouvernementales, est une des principales traductions de ce programme qui propose en fait une extension à l'administration des choses internationales des procédures et recettes mises en place par le système de la public administration à l'échelle domestique entre 1930 et les années 1940. Ce bureau new-yorkais est dirigé par Rowland Egger, le représentant en mission de Public Administration Clearing House en Europe à la fin des années $1930^{44}$, puis par Charles Ascher, qui inaugure alors ses comptes rendus réguliers 
d'activités (les «round-up»). A partir de 1955, alors que les bourses de la Ford sont épuisées, Emmerich essaie vainement de trouver d'autres ressources pour $\mathrm{PACH}$, qui est dissoute en 1957 et délègue ses diverses fonctions aux autres branches du système de la public administration.

Emmerich accepte alors un poste de conseiller (senior consultant) auprès de la Public Administration Branch de la Technical Assistance Administration de l'ONU. C'est à près de 500 personnes, organisation ou journaux aux Etats-Unis et dans le monde qu'il envoie la dépêche de presse annonçant sa nomination, ce qui suggère l'étendue de son réseau et le caractère systématique de son activité de diffusion de l'information ${ }^{45}$. Tout en continuant son travail de conseil en administration internationale (à l'UNESCO en 1963 et 1965 par exemple), il supervise dès lors l'ensemble du travail de recherche des Nations Unies en public administration, dirige le travail d'assistance technique de la section en Amérique Latine et du Sud, et exécute lui-même certaines missions d'assistance auprès des gouvernements de ces pays (notamment au Pérou et au Venezuela). On retrouve dans ses réalisations les recettes éprouvées du 1313 center: changements des techniques et méthodes (organisation, budget, personnel, finances et fiscalité), création d'associations professionnelles d'administrateurs, insistance sur la formation, mise en place d'instituts unissant recherche et enseignement. Au Venezuela, il établit la Comision de Administracion Publica qui est chargée d'étudier la réforme del'Etat vénézuelien à la manière de la Hoover commission aux Etats-Unis ou de la Haldane Commission en Grande Bretagne ${ }^{46}$. Au Pérou, son Instituto Publico de Administracion Publica offre son cours pilote entre novembre 1958 et février 1959, avec des enseignants qui sont des membres de la fonction publique péruvienne dont la quasi totalité a séjourné à l'étranger (Italie, Brésil) avec des bourses du gouvernement des Etats-Unis ou des Nations Unies ${ }^{47}$. Il donne aussi une extension considérable aux programmes de la Escuela Superior de Administracion Publica America Central (ESAPAC), placée sous la direction du Canadien E.P Laberge. L'ESAPAC voit défiler dans ses programmes de formation continue 1586 hauts fonctionnaires des états d'Amérique Centrale entre 1954 à 1966. Ce programme, mené en parallèle avec ceux des organismes états-uniens d'assistance technique (International Cooperation Agency puis Agency for International Development), découle des enquêtes et contacts développés par la Public Administration Clearing House au début des années 1950, et nombre de conseillers techniques américains ou étrangers en public administration employés par les NationsUnies ont transité par le 1313 center, tout comme nombre des contacts d'Emmerich en Amérique Latine. Emmerich quitte les Nations-Unies en 1963 et continue le travail international sous d'autres formes, soit en liaison avec les Nations Unies (pour lesquelles il participe aux divers comités d'évaluation et de programme en public administration), soit par l'enseignement qu'il délivre désormais comme professeur de government and foreign affairs à University of Virginia.

Il s'investit tout particulièrement au sein de l'International Institute of Administrative Sciences. L'institut, basé à Bruxelles, est lié au 1313 center depuis les années 1930, et Emmerich en est le premier président américain de 1962 à 1968. Il y poursuit l'intégration de l'association aux mécanismes de l'assistance technique. En 1962, alors qu'il tente d'obtenir le soutien financier du gouvernement des Etats-Unis à l'Institut, Emmerich explique que ce dernier est devenu progressivement depuis la guerre un "chosen instrument" pour la dissémination des concepts et des pratiques administratives en direction des pays en développement ${ }^{48}$. L'institut reçoit déjà des subventions des Nations Unies et l'US Agency for International Development afin de 
diffuser sa revue dans les pays neufs, subventions qui ont été négociées par Ascher, Emmerich ou Stone qui sont tous trois très présents dans le travail de l'Institut depuis la fin de la Seconde Guerre mondiale. Pendant son mandat, Emmerich accentue l'orientation vers la recherche, vers l'ouverture aux pays neufs et vers la confection d'outils pour les administrateurs des pays du tiers monde. Grâce à ses liens avec le monde philanthropique et avec les Nations Unies, il obtient les soutiens logistiques et financiers d'une telle politique, notamment à travers diverses bourses de la Fondation Ford. L'une d'entre elles finance le projet en administration du développement de l'Institut, qui travaille à l'acclimatation des thèmes de la réforme administrative vers les pays africains, asiatiques et latino-américains à travers un ensemble de séminaires, cours, manuels et bibliographies destinés aux écoles d'administration publique des pays en développement ${ }^{49}$. Il cesse progressivement ses activités en 1969 , et décède en septembre 1970, en ayant consacré toute la deuxième partie de son existence à l'internationalisation de la sphère de la public administration comme conseiller, conspirateur, animateur, administrateur, enseignant ou praticien, particulièrement actif dans la structuration des échanges entre l'Europe et les Amériques.

Donald Stone, une vie de service public ${ }^{50}$

21 Tout célébratif qu'il soit, cette tournure empruntée à une brochure dédiée à Donald Stone est rigoureusement exact. Le curriculum vitae de Stone se parcourt comme un annuaire d'administrations publiques états-uniennes ou internationales, ou d'organisations vouées à l'étude de l'administration publique ${ }^{51}$. Stone est lui aussi un fils d'immigré, puisque c'est son père, anglais, qui vient s'installer au Canada et entreprend des études de pasteur au Canada puis à Oberlin College, une université religieuse très libérale de l'Ohio. En 1913, 10 ans après la naissance de Stone, son père reconverti dans la direction d'une petite entreprise métallurgique qu'il a co-fondée fait lui aussi faillite, tout comme ceux d'Ascher et Emmerich. Sans en tirer de conclusions hâtives, force est de constater que ces aléas de la vie d'entrepreneurs privés les rassemblent. La vie professionnelle de Stone, et une partie de sa vie privée (sa femme Alice est étroitement associée à son activité de savant et d'organisateur), sera en fait placée sous le signe de la public administration, milieu, discipline et savoir dans lesquels il est socialisé très tôt., Donald C.Stone a 22 ans lorsqu'il s'inscrit au Master's program de la School of Citizenship and Public Affairs de Syracuse University à sa sortie de Colgate University (bachelor of sciences, mention science politique et économie). Les conseils de Raymond Fosdick, ancien élève de Colgate, conseiller privé de John D Rockefeller III dans ses affaires philanthropiques et lui même spécialiste de public administration, ne sont peut être pas pour rien dans cette orientation, comme des recommandations ultérieures le laissent penser. Son masters of science in public administration en poche en 1926, le voilà en stage au National Institute of Public Administration à New York (dont Charles Merriam, Leonard White, Raymond Fosdick et Louis Brownlow sont quelques uns des principaux administrateurs). En 1927 on le retrouve assistant au directeur du Cincinnati Bureau of Municipal Research, lequel est le père de l'assistant de Louis Brownlow quand il était directeur des services municipaux (city manager). C'est là qu'il accomplit son initiation aux études (surveys) et aux actions structurelles (installations) qui caractérisent l'application pratique du savoir de la public administration naissante: il s'occupe du règlement du personnel, de l'organisation de l'administration municipale, du système d'évaluation des employés. Il poursuit à Cincinnati une spécialisation dans le travail sur l'organisation administrative de la police, déjà amorcée à New York (et par ailleurs spécialité de Fosdick). C'est cette spécialisation qui lui vaut son 
recrutement en 1928 comme Directeur adjoint du Committee on Uniform Crime Records, une entreprise patronnée par l'International Associations of Chiefs of Police et le Département de la Justice ${ }^{52}$. Ce travail le détourne à la fois d'un séjour en France (Ecole Libre des Sciences Politiques) et des études doctorales qu'il pensait entreprendre avec Charles Merriam à University of Chicago. Mais il lui donne ses lettres de crédit dans le milieu. C'est sur cette base qu'il devient en 1930 directeur de la recherche pour l'International City Manager Association, une des toutes premières organisations professionnelles d'administrateurs à rejoindre le campus de University of Chicago dans le cadre de l'opération lancée par Charles Merriam et ses partenaires. L'ICMA sera le noyau du 1313 center. C'est en son nom que Stone effectue en 1932 son premier voyage d'études en Europe (déjà visitée en 1922 lors d'une école d'été d'économie et de politique de la YMCA), pour y étudier le traitement de diverses questions, du budget au nettoyage des rues en passant par les systèmes nationaux de statistiques de l'activité municipale dans le cadre de son activité de recherche pour le compte de l'association des city managers. C'est aussi là qu'il noue des liens d'amitié avec Charles Ascher et Herbert Emmerich.

Dans cette position, puis à la tête de Public Administration Service, l'agence de conseil et d'ingénierie administrative fondée par les associations du 1313, Stone continue ses investigations de l'administration municipale, bientôt complétées par des travaux analogues sur les administrations des états. Il exécute puis dirige les audits administratifs, la mise en place de nouveaux systèmes de gestion du personnel, de budget ou d'organisation générale dans plus de 100 administrations entre 1933 et 1939 . La croissance des agences fédérales et le soutien du 1313 center à leur conception et mise en place administrative l'amènent à jouer un rôle de conseiller auprès de la plupart d'entre elles, notamment la Public Works Administration. Il est lui aussi aspiré par l'administration fédérale par la suite. En 1939, il est nommé directeur adjoint du nouveau Bureau of Budget. Celui ci est né de la réorganisation de l'exécutif fédéral suite au rapport du President's Committee on Administrative Management (composé de Brownlow, Gulick et Merriam), pour lequel Public Administration Service a effectue de nombreux travaux de recherche. En charge de la division Administrative Management $\mathrm{du}$ Bureau, Stone est pendant neuf ans à la tête de l'organisme qui décide des aspects techniques de l'administration fédérale, depuis les normes en matière d'indemnités de repas ou de formulaires jusqu'aux grandes orientations d'organisation et de management. C'est sous sa houlette que se prépare et fonctionne l'appareil administratif de guerre états-unien.

Assez tôt, comme d'autres administrateurs ou politiciens, et avec ses anciens collègues du 1313 center, Stone se préoccupe de l'organisation administrative du nouvel ordre mondial à naître du conflit. Divers articles et conférences en témoignent, et plus encore son implication effective dans la création des structures des Nations-Unies. Stone est un des participants écoutés de la rédaction de la charte des Nations-Unies, ainsi que des conférences de mise en place de l'organisation, et il siège aux premières assemblées générales en tant que substitut des hommes politiques chargés d'y représenter les Etats-Unis (notamment le sénateur Arthur Vandenberg). Ce rôle est pérennisé par sa nomination au Comité permanent des affaires administratives et budgétaires des Nations Unies. Il a une fonction analogue dans la mise en place administrative de l'UNESCO, et dans les deux organismes se préoccupe non seulement des affaires administratives internes, mais aussi de la mise en place de programmes internationaux 
de recherche et d'assistance en public administration. Ce travail de conseiller, qu'il cumule avec ses responsabilités au Bureau of Budget, ne s'interrompt pas lorsqu'il entre dans l'administration effective du nouvel ordre mondial dans sa version bilatérale (des Etats-Unis vers le reste du monde), en tant que directeur de l'administration pour l'Economic Cooperation Agency/Mutual Security Agency, poste dans lequel il contribue à partir de 1948 à la mise en place du Plan Marshall en Europe et des programmes d'assistance en Asie. Durant toutes ces années, Stone illustre la manière dont les ex-1313ers sont devenus des sages ('wise men') de l'ombre, qui organisent non pas la géopolitique nouvelle comme le font les Acheson, Harriman et Mac Cloy, mais l'administration de cette géopolitique.

Sans rompre avec l'activité de conseil (pour la World Health Organization, la Federal Operations Agency...), Stone semble reprendre son souffle à la direction d'un petite institution d'enseignement supérieur entre 1953 et 1958. C'est cette année là qu'il reprend la route du grand large de l'administration internationale, en tant que doyen et organisateur de la nouvelle Graduate School of Public and International Affaire de University of Pittsburgh. Le projet de l'école, centré autour des questions d'administration, est singularisé par sa dimension internationale, qui imprègne les enseignements (options, enseignants), se retrouve dans le public étudiant qui suitcette formation, et marque l'activité de conseil et de recherche que développent l'équipe rassemblée autour de Stone. Le projet se résume dans l'idée d'enseigner "l'administration du changement" dans les pays en développement, que Stone mentionne à plusieurs reprises dans ses correspondances ${ }^{53}$. Dans le même temps, et en synergie totale avec les objectifs de l'école, Stone intensifie son implication dans le travail de conseil international, à titre personnel et comme représentant de l'institution qu'il dirige. Cela se traduit par une collaboration suivie avec divers organismes gouvernementaux états-uniens (avec une dilection particulière pour le terrain africain sur lequel il effectue diverses missions pour les agences d'assistance technique états-uniennes), soit au sein de l'International Institute of Administrative Sciences. Impliqué dans ce dernier depuis la fin de la Deuxième Guerre mondiale, lorsqu'il en fondait et dirigeait le Committee on Administrative Practices avec le soutien logistique et financier du Bureau of Budget, Stone lance en 1962 la Section on Training Schools and Institutes of administration, à qui une bourse de la Fondation Ford négociée par Emmerich va permettre de devenir le lieu d'échange et de définition des missions et des pratiques pour les divers lieux d'enseignement et de recherche en public administration créés dans les pays en voie de développement avec l'appui des missionnaires en public administration des Nations-Unies ou des programmes bilatéraux états-uniens. C'est aussi dans ce vivier du réseau de l'International Institute of Administrative Sciences que Stone recrute un nombre très important d'enseignants occasionnels et d'étudiants pour l'école de Pittsburgh.

Cette implication internationale se poursuit jusqu'à la fin de la carrière de Stone, qui quitte le décanat de l'Ecole à la fin de la décennie 1960. Il convient de remarquer que, à l'image de certaines dynasties de fonctionnaires internationaux qui se dessinent au $20^{\text {ème }}$ siècle (les Hammarskjöld par exemple), la famille Stone s'inscrit dans cet espace d'activité. Alice Stone milite à haut niveau dans les associations pour les Nations-Unies, et une des filles du couple, avec son époux, s'occupe de développement communautaire (community development) à l'étranger et de recherches en relations internationales. L'interpénétration de la sphère privée et publique du couple renvoie à celle des époux 
Ascher, et semble constituter une ressource essentielle pour permettre d'animer en synergie réseaux professionnels et amitiés familiales.

Conclusion

Les round-ups d'Ascher, dont plusieurs séries nous sont conservées, permettent de saisir les conditions du succès de cette entreprise d'organisation d'un régime circulatoire centré sur la public administration. Ils ont gardé la trace du travail mené au quotidien par Ascher et ses compères. Il y conte ses entreprises, détaille ses rendez vous, note scrupuleusement les noms et les contenus des conversations avec ses interlocuteurs. L'existence de ces documents témoigne du souci de coordination qui marque toute l'entreprise. Ascher rédige ses round ups, à raison d'un ou quatre par mois, afin de tenir régulièrement informés ses compagnons. Les destinataires réguliers en sont Herbert Emmerich, Donald Stone et Rowland Egger, ses complices du 1313. Ces memoranda participent de la logistique de la coopération entre ces membres du noyau dur du système international de la public administration, et complètent leurs fréquentes rencontres, leur correspondance volumineuse et les appels téléphoniques parfois quotidiens au cours desquels ils synchronisent leur action, chacun venant suppléer ou soutenir l'autre dans ses démarches, investigations ou requêtes, avec un redoutable effet de redoublement auprès de leurs interlocuteur. Chaque numéro des round-ups condense en quelques pages toutes les rencontres, démarches, décisions entreprises par Ascher en direction des diverses composantes du réseau que son activité a établie, depuis les conversations aux entractes des spectacles de musique classique des grandes salles new yorkaises jusqu'aux séances de comité de direction des associations dans lesquels il siège, et aux négociations financières avec les organismes gouvernementaux ou philanthropiques. La métaphore de Mickey Mouse se révèle particulièrement pertinente pour saisir ce qui rend possible et efficace cette activité incessante. On est en effet frappé à la lecture des round-ups par l'ubiquité d'Ascher, géographique autant que fonctionnelle. Un jour à Tokyo, le lendemain à Manille puis parcourant le continent européen, Ascher traverse les séances des organes de direction des associations internationales, les conférences des sociétés académiques, les entretiens au Département d'Etat ou les sessions des commissions d'attribution des bourses Fulbright avec une ligne directrice constante : la coordination de l'ensemble de ces activités au service de la généralisation des méthodes d'action et d'organisation de la public administration. Juge un jour, il est parti le lendemain, il crée la demande et assure l'offre, comme dans le cas d'école exposé dans la citation qui débute cette note.

Cette ubiquité est partagée par ses compagnons, à l'image de Price, Emmerich, Stone ou Egger. Tous l'accompagnent d'un multipositionnement professionnel, occupant successivement ou simultanément des positions universitaires, administratives et associatives, à cheval sur l'espace domestique et le monde 'entre les nations', tout en alternant entre les rôle de conseiller, d'expert ou d'entrepreneurs dans tous ces cénacles. Cette ubiquité n'est pas pour autant versatile; elle se traduit chez Ascher en efficacité grâce à la tenue méticuleuse et quasi-obsessionnelle de notes, agendas et carnets où sont consignés les noms de tous ceux qu'il rencontre, les propos échangés aussi bien que les moyens de transport utilisés ou les scores des matchs de tennis ou de ping pong joués avec son épouse. La sociabilité du couple tourne d'ailleurs autour du travail international, et la maison Ascher est un lieu de sociabilité gastronomique et d'hébergement convivial pour un nombre croissant de visiteurs étrangers qui rejoignent le système international de la public administration ${ }^{54}$. Cette activité d'interaction directe s'accompagne par ailleurs d'une correspondance abondante, qui 
mêle la chaleur des relations personnelles aux directives et suggestions concernant la marche du système $e^{55}$.

Fidèles à leur acculturation dans le système domestique créé par Louis Brownlow au 1313 pour la rénovation des modes de gouvernement et d'administration dans les EtatsUnis des années 1930, Ascher et ses complices cultivent sur la scène internationale l'effacement, le désintéressement, la relation amicale, la mise en contact, la suggestion, refusent les positions de pouvoir auquel ils préfèrent les situations de conseil, se livrent rarement à des démonstrations d'autorité et sont par dessus tout soucieux de la dénationalisation de leur action. Tant par lucidité que par conviction, et dans un mélange souvent paradoxal, ils parlent et agissent au nom de l'universel qu'ils tentent d'édifier. Que cet universel soit fortement teinté des valeurs états-uniennes et que sa réalisation signifie en particulier la diffusion, en matière de public administration, de certaines des manières de faire qu'ils ont eux-mêmes contribué à mettre en place dans leur pays, cela ne fait aucun doute pour eux. Cependant, leur travail est bien celui de la proposition, soucieuse de traduction et d'adaptation par les partenaires de l'entreprise d'internationalisation, et non pas celui de la domination. La dissociation coopérative dont ils témoignent vis à vis des structures étatiques états-uniennes et de leur action en matière d'assistance technique en public administration témoigne d'ailleurs d'une distance relative dont il faut tenir compte dans l'analyse : leur science politique, leur science de l'administration publique, n'est pas une science d'Etat au sens où le droit, la science politique et les "sciences administratives" continentales peuvent l'être, renvoyant ainsi bien évidemment à l'histoire de la constitution des disciplines académiques et des structurations de la vie publique aux Etats-Unis. Leur action ne se résume pas à une exportation des principes d'une public administration " états-unienne " en direction $d u$ reste $d u$ monde. Tout d'abord, il est patent que cette public administration domestique a été définie (comme communauté, discipline, corpus théorique, pratique) par un travail d'importation, de traduction et d'appropriation d'expériences européennes, en un processus commencé à la fin du $19^{\text {ème }}$ siècle et parachevé par le travail du 1313 center. Ensuite, le travail d'internationalisation mené dans les décennies qui suivent la Seconde Guerre Mondiale concerne aussi le terrain états-unien, qu'Ascher et ses compagnons sont soucieux d'irriguer par la circulation des informations sur les innovations administratives et gouvernementales étrangères. Parce que leur localisation, leur travail, leurs valeurs se situent entre impérialisme et pluralisme, entre l'inscription dans un cadre national et le dépassement de celui-ci pour un "entre-monde » au delà -ou en deça- des états-nations ${ }^{56}$, travailler sur ces " little wise men " est une contribution au travail d'internationalisation entendu comme processus universalisant. Les suivre dans les oscillations de leur inscription spatiale, institutionnelle et professionnelle permet de tenir ensemble un souci critique, (en prêtant donc attention aux «impérialismes de l'universel $»^{57}$ ou à ce que l'internationalisation doit aux structurations nationales) et la prise en compte des effets universalisants et pluralistes de ces heurts et affrontements (et donc en prenant au sérieux les dispositions 'transnationales' que contient, entre autres, l'universalisme états-unien, tel qu'exprimé au début du $20^{\text {ème }}$ siècle dans les proclamations de Woodrow Wilson, les confessions de Randolph Bourne ou les anticipations du britannique William Stead). Le travail avec les notions d'impérialisme ou de domination se complexifie dès lors des notions de fertilisation croisée, appropriation, imitation, en même temps que la considération des processus d'internationalisation et de leur lecture en rivalités et positions nationales se double d'une prise en compte de projets spécifiquement 
transnationaux, qui dessinent un espace d'action, de pratique, d'enjeux qui se situe là encore au delà ou en deçà des échanges entre nations ou entre individus et collectifs nationalement identifiés. C'est cette voie qui peut être fructueuse pour une contribution à l'historicisation de ce que par raccourci on appelle la globalisation (l'histoire des phénomènes d'interconnexion et d'interdépendance), en rejoignant les propositions qui appellent à l'histoire des processus d'internationalisation sous différentes espèces, et en prêtant une attention particulière aux stratégies, projets et menus travaux d'universalisation qu'ils permettent d'identifier ${ }^{58}$. Passer pour cela par l'étude des régimes et des structures permet de proposer des pistes complémentaires pour étudier les 'conditions sociales de circulation internationale des idées'. Dans un article souvent cité et portant ce titre ${ }^{59}$, Pierre Bourdieu insistait sur la manière dont la circulation est liée aux rapports de force et aux usages dans les espaces savants ou politiques nationaux. A sa suite, Yves Dezalay et d'autres ont fait de ce principe le moteur de leur réflexion sur "l'internationale des élites nationales » ${ }^{60}$, et ont insisté avec bonheur sur le rôle matriciel des champs nationaux. Cet article est plus attentif à la manière dont des acteurs et des institutions se dessinent un espace transnational de manœuvre tendant à l'autonomie par rapport aux espaces nationaux. A terme, ces deux angles me semblent diverger sur les types de trajectoire et d'usages étudiés : d'un côté on se situe plus en termes d'usages stratégiques de ressources transnationales pour des fins nationales, et on privilégie une logique d'échelle, d'espaces emboîtés. De l'autre, on est plus dans une logique de trajectoires d'individus, d'organisations, d'institutions, de projets qui traversent différents espaces de pratiques (et les constituent/reconstituent au passage), l'utilisation et le cumul de ressources se faisant simultanément dans ces différents espaces sans que l'un soit premier. Mais tous deux contribuent à une histoire en cours d'élaboration des "luttes de l'universel», dont les initiatives et acteurs en provenance des Etats-Unis d'Amérique sont des protagonistes majeurs pour l'histoire contemporaine.

Liste des abréviations :

NAHO : National Association of Housing Officials

PACH : Public Administration Clearing House

ASPA : American Society of Public Administration

IULA : International Union of Local Authorities

IIAS : International Institute of Administrative Sciences

IFHTP : International Federation of Housing and Town Planning

ICA : International Cooperation Agency, Federal Government of the United States oif America

ECOSOC : Economic and Social Council, United Nations

NGOs : Non Governmental Organizations

IGOs : Inter Governmental Organizations 


\section{NOTES}

1. The Wise men: six friends and the world they made, New-York: Simon \& Schuster, 1986.

2. Quelques exemples avec Joel Best, ed., How Claims Spread: Cross-National Diffusion of Social Problems, New York : Aldine de Gruyter, 2001 ; Janine Wedel, Collision and Collusion : The Strange Case of Western Aid to Eastern Europe, 1989-1998, New York : St. Martin's Press, 1998 ; Richard Rose, Lesson-Drawing in Public Policy : A Guide to Learning Across Time and Space, Chatham : Chatham House, 1993 ; Wade Jacoby, Imitation and Politics : Redesigning Modern Germany, Ithaca : Cornell University Press, 2000.

3. A noter toutefois que celui ci n'était pas auparavant hermétique, cf. Richard Vidmer \& James Thompson, Administrative sciences and politics in the USSR and the United States. Soviet responses to American management techniques 1917- present, New York : Praeger, 1983.

4. Cet article développe et étend quelques un des points abordés dans Pierre-Yves Saunier, «Tel Mickey Mouse jouant au tennis... Note de recherche : Charles S.Ascher et l'internationalisation de la public administration ", Actes de la Recherche en Sciences Sociales, 151-152 (mars 2004), 49-56.

5. Peri E. Arnold, Making the managerial presidency : comprehensive reorganization planning 1905-1980, Princeton : Princeton University Press, 1986.

6. Quelques étapes de ce débat avec Stephen Skowronek, Building a new American state : the expansion of national administrative capacities, 1877-1920, New York : Cambridge University Press, 1982 ; Louis Galambos, ed., The New American state : bureaucracies and policies since World War II, Baltimore : Johns Hopkins University Press, 1987 ; Daniel P.Carpenter, The forging of bureaucratic autonomy : reputations, networks, and policy innovation in executive agencies, 1862-1928, Princeton, N.J. : Princeton University Press, 2001. Voir aussi le vif et rapide panorama offert par Brian Balogh dans « The state of the State among historians », Social Science History, 27, 30 (2003), 455-463.

7. Woodrow Wilson, « The study of administration », Political science quarterly $56: 2$ (1887) ; Frank Goodnow, Politics and administration, New-York : Macmillan, 1900.

8. On trouvera des matériaux sur ce point dans l'historiographie sur l'ère progressiste, chez Allen Davis, Spearheads for reform. The social settlements and the progressive movement 1890-1914, New York : Oxford University Press, 1967 ; Robert Wiebe, Business and reform : a study of the progressive movement, Cambridge : Harvard University Press, 1962 et The search for order 1877-1920, New-York : Hill \& Wang, 1967 ; Martin Schiesl, The politics of efficiency: municipal administration and reform in America 1880-1920, Berkeley : University Of California Press, 1977 ; Ellis Hawley, The great war and the search for a modern order, New-York : Saint Martin's Press, 1992.

9. Jonathan Kahn, Budgeting democracy. State building and citizenship in America 1890-1928, Ithaca : Cornell University Press, 1997.

10. Les travaux de Barry Karl, Executive reorganization and reform in the New Deal, Cambridge : Harvard University Press, 1963 et Charles E. Merriam and the study of politics, Chicago : University of Chicago Press, 1974, ont été les premiers à mettre en évidence cette conjonction et ses effets. Il faut noter à ce propos que Karl, qui commença de travailler sur ces sujets lors de ses études à la Graduate School of Public Administration (Harvard), collabora étroitement avec Louis Brownlow pour la rédaction de son 
autobiographie parue en 1958, et fut un correspondant d'Herbert Emmerich à cette occasion.

11. Pour une approche plus détaillée, Pierre-Yves Saunier, « Ulysses of Chicago :

American Foundations and public administration 1900-1960 », dans Giuliana Gemelli \& Roy Mc Leod, American Foundations in Europe. Grant-giving policies, cultural diplomacy and transatlantic relations 1920-1980, Bruxelles : Peter Lang, 2003, 115-128 ; « Administrer le monde ? Les fondations philanthropiques et la public administration aux Etats-Unis ", Revue Française de Science Politique, 53 : avril 2003, 237-255.

12. Peri E. Arnold, Making the managerial presidency : comprehensive reorganization planning 1905-1980, Princeton : Princeton University Press, 1986.

13. L'histoire complète du service a été écrite par Larry Berman, The office of management and budget and the presidency 1921-79, Princeton : Princeton University Press, 1979.

14. Le Council of Intergovernmental Relations, qui œuvre à cette intégration, est d'ailleurs une des associations du 1313 Center, financée par le Spelman Fund of New York. Harold Smith en sera un des principaux animateurs. Sur cette intégration, voir Daniel J. Elazar, « The shaping of intergovernmental relations in the 20th century ", Annals of the American Academy of Political Science, 359 (1965), 10-22. Un exemple pratique avec Philip J. Funigiello, The challenge to urban liberalism Federal-City relations during World War II, Knoxville : The University of Tennessee Press, 1978.

15. Sur cette longue durée, voir notamment Michel Sennelart, Les arts de gouverner. Du régime médiéval au concept de gouvernement, Paris : Seuil, 1995 ; Michael Stolleis, Histoire du droit public en Allemagne. Droit public impérial et science de la police 1600-1800, Paris : PUF, 1998 ; Pierangelo Schiera Von, der Verfassung zur Verwaltung : burgerliche Staatswissenschaft in Deutschland und Italien nach der nationalen einigung, Francfort : Klostermann, 1984.

16. Voir les numéros spéciaux Sciences du Politique (Genèses, 37, décembre 1999), Les savants et le politique (Politix, 48, $4^{\circ}$ trimestre 1999), Sur la science de l'Etat (Actes de la recherche en sciences sociales, 133, juin 2000), Sur la formation des sciences de gouvernement (Revue française de science politique, 53, 2, avril 2003), ou les travaux du groupe Sciences camérales en Allemagne et en France dirigé par Pascale Laborier. Soulignons ici que ces préoccupations ne s'adressent pas qu'au gouvernement « national ».

17. André Mater, « Nature et classification des faits administratifs », L'Année administrative 1 (1903), 339.

18. Charles Beard, " The role of administration in Government ", The work unit in Federal Administration (1937), 3 (ma traduction).

19. Cf. par exemple Odile Henry, « De la sociologie comme technologie sociale. La contribution de Jean Coutrot 1895-1941 », Actes de la recherche en sciences sociales, 153 (2004), 48-64.

20. On pense ici à certains tayloristes états-uniens comme Morris Cooke, les groupements qui suivent Fayol ou les 'documentalistes' tels que les Néerlandais de la Nederlansche Vereeniging van Gemeente Belangen qui insistent sur la classification et la systématisation des documents administratifs.

21. Cette approche est particulièrement développée aux Etats-Unis. Voir par exemple la Wisconsin idea, ce moment où la conception et l'exécution des politiques publiques de l'état du Wisconsin s'appuie sur les ressources de l'University of Wisconsin, au mouvement de réforme municipale (municipal reform) des années 1890-1910 qui s'arc 
boute sur les enseignants de science politique, de sociologie, d'économie ou de droit, ou au développement, dans les années 1920, de la discipline de la public administration à partir des paradigmes et des organisations des sciences sociales.

22. Cette approche par le droit administratif et constitutionnel, qui mobilise les juristes des institutions de l'Etat (conseils d'Etat notamment) et les professeurs de Droit semble particulièrement développée dans certains milieux continentaux, notamment en France, en Belgique ou en Espagne. La notion de « sciences administratives » est particulièrement liée à cette approche.

23. Un des terrains privilégiés de la problématisation scientifique des faits de gouvernement et d'administration est la municipalité et plus particulièrement la municipalité urbaine, point d'attaque pour des positions de « perfectionnement » et de « réforme » des méthodes et pratiques politico administratives dans divers contextes (Cf. Renaud Payre, «À la recherche de la 'science communale'. Les 'mondes' de la réforme municipale dans la France de la première moitié du vingtième siècle », Thèse de doctorat de science politique, Université Pierre Mendès France Grenoble II, décembre 2002 ; Federico Lucarini, Scienze comunali e pratiche di governo in Italia (1890-1915), Milan : Giuffré, 2003 ; Michael Frisch, « Urban theorists, urban reform and American political culture in the Progressive period », Political Science Quarterly, $97: 2$ (1982), 295-315.

24. Nicolas Guilhot, « Les professionnels de la démocratie : logiques militantes et logiques savantes dans le nouvel internationalisme américain ", Actes de la recherche en Sciences Sociales, 139 (2001), 53-65.

25. Je pense ici à l'urbanisme, à l'hygiène, au logement et à d'autres éléments constitutifs de l'idiome de la réforme. Quoique j'exagère sans doute ici les positions de l'auteur, attaché à considérer la nationalisation des mondes réformateurs et à questionner la profondeur de leur mise en scène internationale, le travail de Christian Topalov sur la catégorie du chômage me semble très parlant sur la manière dont des problématiques nationales sont puissamment informées par des discussions internationales ( From the social question to urban problems : reformers and the working classes at the turn of the 20th century ", International Social Science Journal, 42, 3 (125), 1990, 319-33 ; Naissance du chômeur 1880-1910, Paris : Albin Michel, 1994.

26. Sur l'Institut, voir Renaud Payre, « L'espace des circulations. Réseaux d'acteurs et fabrique transnationale des sciences administratives (années 1910-années 1950) », dans Frédéric Audren et al., dir, Les sciences camérales. Paris : PUF, à paraître.

27. Un très beau cas est fourni par l'entreprise de grand envergure du gouvernement du Japon au moment du Meiji, cf. Eleanor Westney, Imitation and Invention. The Transfer of Western Organizational Patterns to Meiji Japan, Cambridge : Harvard University Press, 1987.

28. Le cabinet Arthur Young \& Co se voit proposer en 1918 de remodeler la fonction publique canadienne juste avant que Edwin Griffenhagen et ses hommes, fraîchement organisés en société indépendante de Young \& $\mathrm{Co}$, n'interviennent dans l'administration municipale montréalaise. Alasdair Roberts, So-called experts. How American consultants remade the Canadian civil service 1918-1921, Toronto : Institut d'Administration Publique du Canada, 1996 ; Michèle Dagenais, Des pouvoirs et des hommes, Montréal : Institut d'Administration Publique du Canada/McGill-Queen's University, 2000, 56 et suivantes.

29. Jusqu'à présent, on a plutôt insisté sur le développement par la Ford des écoles de gestion et de management (Cf. Giuliana Gemelli, From imitation to competitive- 
cooperation: Ford Foundation and management education in Western Europe (1950s-1970s), Florence : European University Institute, 1997), mais cette facette publique est tout aussi importante.

30. Les travaux sur la ré-appropriation française des procédures de comptabilité nationale dans les années 1940-50, puis de l'outil du Program Planning and Budgeting System (PPBS) développé au sein de la RAND Corporation dans les années 1960, suggèrent des pistes intéressantes en la matière. Cf. François Fourquet, Les Comptes de la Puissance, Paris : Encres, 1981 et Vincent Spenlehauer « Intelligence Gouvernementale et Sciences Sociales », Politix, 48, 1999, 95-128.

31. Pour une présentation d'ensemble, Pierre-Yves Saunier, «Sketches from the Urban Internationale. Voluntary societies, international organizations and US Foundations at the city's bedside 1900-1960 ", International Journal for Urban and Regional Research, 25 : 2 (juin 2001), 380-403.

32. University of Virginia Special Collections Library, Herbert Emmerich Papers, RG 21/38.711, box 3, Folder Charles S. Ascher 1956-1962, « Round up 1958 \#2, February 1-28 $1958 », 1$ (ma traduction).

33. Voir Pascale Casanova, La République mondiale des lettres, Paris : Le Seuil, 1999, ainsi que son article «Consécration et accumulation du capital littéraire. La traduction comme échange inégal », Actes de la recherche en sciences sociales, 144 (septembre 2002), 7-21.

34. Armand Mattelart, Histoire de l'utopie planétaire. De la cité prophétique à la société globale, Paris : La Découverte, 2000.

35. D'abord avocat dans deux firmes de Wall Street, Ascher rejoint en 1926 la City Housing Corporation pour être son conseiller juridique et secrétaire. Son travail est marqué par sa collaboration à l'entreprise urbanistique et immobilière de Radburn, «the city of the motor age ", icône de l'urbanisme états-unien de l'entre deux guerres. Dans cette communauté du New Jersey construite et gérée par la City Housing Corporation, Ascher travaille aux côtés de ses futurs partenaires Herbert Emmerich et Louis Brownlow. Chargé du suivi légal de tout ce qui touche aux questions de l'immobilier, de l'urbanisme et des procédures de gouvernement dans la nouvelle communauté, il est identifié par Evan McKenzie comme un des inventeurs des formes de gouvernements privés qui régissent aujourd'hui certaines des formes résidentielles et communautaires connues sous le nom de gated communities (Privatopia. Homeowner associations and the rise of residential private governments, New Haven : Yale University Press, 1991).

36. Brownlow fait de l'expression le titre du second volume de son autobiographie, The autobiography of Louis Brownlow, Second half. A passion for anonymity, Chicago : University of Chicago Press, 1958.

37. On peut mentionner ici que Charles Ascher présentait quelques dispositions biographiques pour opérer en dehors de l'espace domestique états-unien. Il fait partie d'une famille juive allemande immigrée, est un ancien élève de l'Ethical Culture School de New York dirigée par Felix Adler, parle français et allemand depuis son enfance. De surcroît, il a étudié dans une université et un département fortement marqués par certains personnalités tournées vers le travail transnational, qu'il s'agisse de Nicholas Butler, président de Columbia, figure du 'pacifisme' réaliste des juristes incarné par le mouvement d'arbitrage des conflits et dirigeant du Carnegie Endowment for International Peace, ou de James Shotwell, lui aussi fortement impliqué dans le mouvement pacifiste puis dans le soutien aux organismes intergouvernementaux nés du traité de Versailles. 
Cf. Columbia University, Oral History Collection, Charles Ascher interview (19 sessions de 1969 à 1971)

38. Pour une formulation des espaces temps du projet et de ses catégories (« urgence ", « hémisphérique » et « global »), on peut se reporter, par exemple, au mémorandum rédigé par Louis Brownlow, Charles Merriam et Guy Moffett (secrétaire exécutif du Spelman Fund of New York) pour servir de plan d'action à Nelson Rockefeller lorsqu'il devient Coordinator for Inter American Affairs en 1940 (UVA, Guy Moffett papers, Mss 9768, box 3, Folder Latin America 1940-42, Memorandum, notamment p. 1-8).

39. Le lieu n'est pas indifférent à cause de la propension de Félix Adler, fondateur de l'école à développer son entreprise éthique au delà de l'espace états-unien comme en témoignent ses multiples initiatives dont le Universal Races Congress de 1911 à Londres. Cf. les travaux de Tracie Matysik dont « Internationalist activism and global civil society at the high point of nationalism : the paradox of the universal races congress 1911 ", dans Anthony Hopkins, ed, Global history. Interactions between the universal and the local, New York : Palgrave, 2006.

40. Selon son bras droit Don Price, Ford Foundation Archives, Oral History Project, Interview with Don K Price, juin 1972, 46

41. University of Virginia, Herbert Emmerich Papers, RG 21/38.711, box 26, folder « 1945-1963 UNESCO ». Le journal qu'Emmerich a tenu durant la conférence préparatoire de l'UNESCO à Londres en novembre 1945 est particulièrement intéressant parce qu'il documente le fonctionnement interne de la délégation états-unienne et son rôle dans la conférence.

42. Ford Foundation Archives, reel 2472 Grant 62-43, reel 1026 Grant PA 50-6 et Grant PA 52-144.

43. 45 millions de dollars 2005 selon le site www.measuringworth.com des économistes Samuel Williamson et Lawrence Officer (méthode de calcul au prorata du Produit National Brut).

44. Pierre-Yves Saunier, "Selling the idea of cooperation. The US Foundations and the European components of the Urban Internationale (1920s-1960s) », dans Giuliana GEMELLI, dir., American Foundations and large scale research : construction and transfer of knowledge, Bologna : Clueb, 2001, 219-246. Aussi disponible sur http:// archive.rockefeller.edu/publications/conferences/saunier.pdf

45. University of Virginia, Herbert Emmerich Papers, RG 21/38.711, box 25, Folder « UN Herbert Emmerich fan letters on appointment »

46. University of Virginia, Herbert Emmerich Papers, RG 21/38.711, box 25, Folder «1956-64 UN Venezuela ».

47. University of Virginia, Herbert Emmerich Papers, RG 21/38.711, box 25, Folder « 1957-1959 UN-Peruvian mission ».

48. University of Virginia, Herbert Emmerich Papers, RG 21/38.711, box 16, Folder " IIAS US government ", A proposal for United States government membership in the International Institute of Administrative Sciences, 1962, 2.

49. University of Virginia, Herbert Emmerich Papers, RG 21/38.711, boxes 9 et 14 . 50. A life time of public service. A tribute to Donald Stone, New-York : American Society of Public Administration, 1996. Le parcours de Stone est ici retracé à l'aide de divers fonds d'archives conservés à University of Chicago (Charles Merriam Papers, Presidents Papers, Louis Brownlow diaries), à University of Virginia (Herbert Emmerich Papers, Rowland Egger Papers), à Columbia University (Charles Ascher papers) et au 
Rockefeller Archive Center de Tarrytown. Les papiers personnels de Donald Stone, à Pittsburgh University, n'ont pas encore pu être explorés.

51. Jusqu'aux emplois estivaux de son époque estudiantine, consacrés à des travaux pour les opérations de lotissement d'une mairie de l'ohio, état dont il est originaire. 52. Ce Comité va entre autres donner naissance au fichier national des crimes et des délits, base de l'action du nouveau Federal Bureau of Investigation.

53. Cette orientation internationale se retrouve au même moment dans d'autres cursus (enseignements, bourses, recrutement), eux aussi appuyés par les grandes fondations philanthropiques, notamment à la Graduate School of Public Administration de Harvard University dont l'ancien 1313er Don Price reçoit la direction la même année. 54. On peut souligner que cette confusion entre vie privée et vie publique est facilitée par l'activité d'Helen Ascher. Militante de l'American Union for Civil Liberties dans les années 1920, un groupement bâti autour de la défense de la liberté d'expression, Helen Ascher participe elle aussi au travail international dans les années 1950. Elle représente la Word Federation for Mental Health auprès de l'ONU et préside diverses organisations d'ONG.

55. Ascher est, par exemple le grand ordonnateur des lettres d'anniversaire aux grands anciens du système international de la public administration, ou encore des lettres de condoléances en hommage aux disparus, occasions pour lesquelles il déclenche de véritables vagues de courriers individuels ou institutionnels.

56. Le terme d'entre-monde est une traduction libre de l'expression 'world in between' proposée par Daniel Rodgers dans Atlantic crossings. Social politics in a progressive age, Cambridge : Harvard University Press, 1998.

57. Pierre Bourdieu « Deux impérialismes de l'universel » dans Tom Bishop et Christine Fauré, dir., L'Amérique des Français, Paris : Editions François Bourin, 1992, 149-155.

58. Sur cette proposition d'historicisation, voir par exemple Eric Foner, « American Freedom in a Global Age », The American Historical Review, 106 : 1 (2001), 1-16 ; Frederick Cooper, "What is the concept of globalization good for ? an African Historian's perspective », African affairs, 100 (2001), 189-213 ou dans une perspective plus réduite Pierre-Yves Saunier, "Taking up the bet on connections : a municipal contribution », Contemporary European History, $11: 4$ (2002), 507-527.

59. Pierre Bourdieu, « Les conditions sociales de la circulation internationale des idées ", Romanistiche Zeitschrift für Litteraturgeschichte/Cahiers d'histoire des littératures romanes, XIV, 1-2 (1990), 1-10.

60. Cf. ses introductions et contributions au numéros Sociologie de la mondialisation et Les ruses de la raison impérialiste des Actes de la Recherche en Sciences Sociales.

\section{RÉSUMÉS}

Cet article traite du travail transnational mené entre les années 1940 et les années 1960 par divers protagonistes de public administration états-unienne (entendue ici comme discipline, pratique, théorie et communauté professionnelle). Ce travail se développe entre organismes intergouvernementaux, associations internationales et sociétés professionnelles états-uniennes. 
On propose ici de contribuer à l'historicisation des phénomènes et processus d'internationalisation en passant par les pratiques qui les définissent, les fondent et les supportent, à travers l'activité de trois figures états-uniennes de la public administration, saisis dans leur activité quotidienne de "traducteurs de l'universel », occupés à développer un projet universaliste et universalisant, qui postule l'existence de phénomènes identifiables comme relevant de l'activité d'administration et susceptibles d'être appréhendés d'une semblable manière scientifique quel que soit leur contexte.

This article examines the transnational endeavors that presided over the establishment and maintenance of a structured regime of circulation in public administration considered as a profession, a theory, a discipline and a governmental practice. The three individuals who are at the core of the article were vital in organizing this regime, and their contribution to integrate the contribution of inter-governmental organizations, non governmental organizations, professional US societies and US universities has left a deep imprint in the field. Assessing their contribution is the opportunity to develop some hunches and hypothesis on the existence of "universalizing projects" that have shaped the interconnection and interdependency of our modern world.

\section{INDEX}

Mots-clés : administration, fondations philanthropiques, organisations non gouvernementales, universel

Keywords : transnational, public administration, philanthropic foundations, non governmental organisations, universal

\section{AUTEUR}

\section{PIERRE-YVES SAUNIER}

Chargé de recherches au CNRS, UMR 5600 Environnement Ville Société, Lyon, pierreyves.saunier@wanadoo.fr 